\title{
Spectrum of cardiovascular diseases in six main referral hospitals of Ethiopia
}

\author{
Dejuma Yadeta, ${ }^{1}$ Senbeta Guteta, ${ }^{1}$ Bekele Alemayehu, ${ }^{1}$ Dufera Mekonnen, ${ }^{1}$ \\ Etsegenet Gedlu, ${ }^{2}$ Henock Benti, ${ }^{3}$ Hagazi Tesfaye, ${ }^{4}$ Samuel Berhane, ${ }^{4}$ \\ Abraha Hailu, ${ }^{4}$ Abadi Luel, ${ }^{5}$ Tedros Hailu, ${ }^{5}$ Wandimu Daniel, ${ }^{6}$ Abraham Haileamlak, ${ }^{6}$ \\ Esayas Kebede Gudina, ${ }^{7}$ Gari Negeri, ${ }^{7}$ Desalew Mekonnen, ${ }^{8}$ Kindie Woubeshet, ${ }^{9}$ \\ Tariku Egeno, ${ }^{9}$ Kinfe Lemma, ${ }^{9}$ Vibhu R Kshettry, ${ }^{10}$ Endale Tefera ${ }^{2}$
}

For numbered affiliations see end of article.

\section{Correspondence to}

Dr Dejuma Yadeta, Department of Internal Medicine, School of Medicine, Addis Ababa University, Addis Ababa 58052, Ethiopia; dejuya@yahoo.com

Received 29 August 2016 Revised 10 April 2017 Accepted 18 April 2017

\section{CrossMark}

To cite: Yadeta $\mathrm{D}$, Guteta S, Alemayehu B, et al. Heart Asia

2017;9:1-5.. doi:10.1136/ heartasia-2016-010829

\begin{abstract}
Background The spectrum of cardiovascular diseases varies between and within countries, depending on the stage of epidemiological transition and risk factor profiles. Understanding this spectrum requires regional and national data for each region or country. This study was designed to determine the spectrum of cardiovascular diseases in six university hospitals in Ethiopia.
\end{abstract}

Methods This is a cross-sectional study of the spectrum of cardiovascular diseases in six main referral/teaching hospitals located in different parts of the country. Consecutive patients visiting the follow-up cardiac clinics of these hospitals from 1 January to 30 June 2015 were included in the study. Data were collected on a pretested questionnaire.

Results A total of 6275 patients (58.5\% females) were included in the study. Nearly $61 \%$ of the patients were from urban areas. The median age was 33 years (IQR 14-55 years). Valvular heart disease was the most common diagnosis, accounting for $40.5 \%$ of the cases. Of 2541 patents with valvular heart disease, 2184 (86\%) were cases of chronic rheumatic heart disease.

Conclusion Our study shows that chronic rheumatic valvular heart disease is the most common cardiovascular diagnosis among patients seen at cardiology clinics of six referral/teaching hospitals in the country, followed by congenital heart diseases. Hypertensive and ischaemic heart diseases also accounted for a significant proportion of the cases. Therefore, strategies directed towards primary and secondary prevention of acute rheumatic fever as well as prevention of risk factors for hypertension and ischaemic heart disease may need to be strengthened.

\section{BACKGROUND}

In the last few decades, developing countries have been undergoing an epidemiological transition in which the causes of morbidity and mortality are shifting from primarily communicable to primarily non-communicable diseases, including cardiovascular diseases. ${ }^{1-4}$ The spectrum of cardiovascular diseases varies between, and within, countries depending on the stage of epidemiological transition and risk factor profiles ${ }^{56}$ necessitating regional or national data for each region or country. Knowledge of the full spectrum of cardiovascular diseases will help implement research programmes, advocacy, and education. ${ }^{7}$ Reassessment and implementation of public health policy in countries in epidemiologic transition requires evidence-based decision making.

Ethiopia is the second most populous country and one of the fastest growing economies in Africa. However, there are few hospital-based studies on the spectrum of cardiovascular diseases addressing just one or two segments of this important problem. Therefore, our study is designed to determine the spectrum of cardiovascular diseases in six university hospitals located in different parts of the country.

\section{METHODS}

This is a cross-sectional study of the pattern of cardiovascular diseases in six university/referral hospitals located in different parts of the country where the majority of aetiologic diagnoses of cardiovascular diseases are made. The study was conducted in Addis Ababa university hospital (Tikur Anbessa Specialised Hospital, TASH), Mekele university hospital (Ayder hospital), Gondar university hospital, Jimma university hospital, Saint Paul Medical College hospital, and Hawassa university hospital (figure 1). All of the hospitals included in this study have separate paediatric and adult cardiology clinics. The paediatric cardiac clinics generally serve patients with congenital and acquired cardiac diseases who are younger than 18 years of age.

The adult and paediatric cardiologists trained nurses working in the cardiac clinics to enter data from the patients and their records to the data questionnaires. These trained nurses sat in the cardiac clinics and entered data while the physicians/investigators were seeing patients. After completion of the questionnaires, physicians reviewed them for accuracy and completeness. The adult and paediatric cardiologists then checked all completed questionnaires once again before entering the data into the database. Consecutive patients visiting the follow-up cardiac clinics of the above mentioned hospitals from 1 January 2015 to 30 June 2015 were included in the study. Patients admitted to the inpatient facilities during the study period were also included in the study since they were referred to follow-up clinics after discharge. Diagnosis was established in all patients by transthoracic echocardiography undertaken by paediatric or adult cardiologists or internists and paediatricians trained in echocardiography and with good experience of 


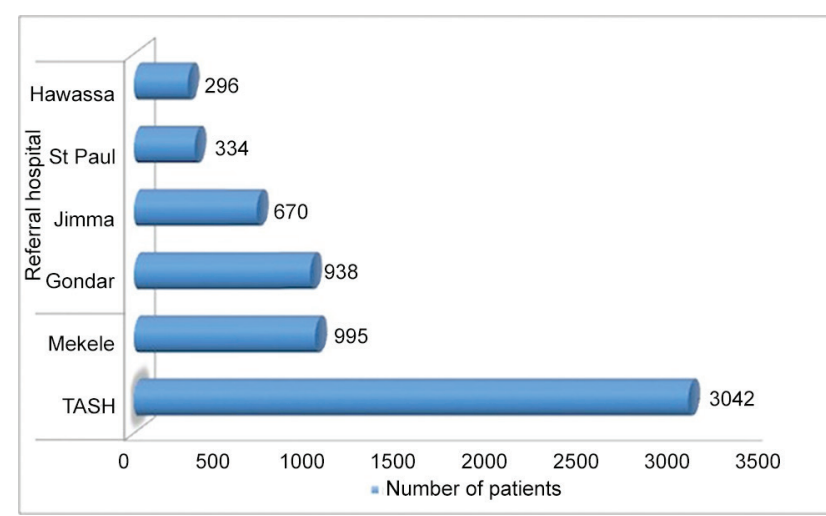

Figure 1 Number of cases included from each referral hospital. TASH: Tikur Anbessa Specialised Hospital.

the technique. Patients with no echocardiographic diagnosis were excluded from the study.

Diagnosis of chronic rheumatic heart disease was based on the World Heart Federation echocardiographic criteria. ${ }^{9}$

Ischaemic heart disease included three entities:

1. Angina: Patients with chest pain and a high (>90\%) pretest probability of coronary artery disease (CAD) and patients with chest pain plus an intermediate (10$90 \%)$ pretest probability with a positive stress test were included. The value of stress testing for the diagnosis of CAD is in the intermediate risk patients (10-90\% pretest probability of CAD). ${ }^{10}$ Since stress testing is not available in most public hospitals where the study was done we may underestimate the true prevalence of ischaemic heart disease

2. Acute myocardial infarction was defined by the presence of elevated high sensitivity troponin together with acute onset chest pain, and/or typical ECG changes.

3. Prior myocardial infarction including patients who present with or without heart failure in whom echocardiography detected regional wall motion abnormality in the absence of a history of acute coronary syndrome. For definition of this kind of ischaemic heart disease we relied on the WHO 2008-9 revision category B definition due to lack of reports of cardiac biomarkers and coronary angiography in our centres across the country. ${ }^{11}$ To increase the specificity of echocardiography, the risk profile of the patients (such as age, presence of coronary risk factors) and/or pathologic Q waves on ECG were used to predict the possibility of ischaemia as a cause of the regional wall motion abnormality in a dilated left ventricle with reduced ejection fraction. This definition and the criteria have also been used in similar resource constrained settings. ${ }^{12}$ We acknowledge that regional wall motion abnormality on echocardiography has limitations in reliably differentiating ischaemic heart disease from dilated cardiomyopathy, particularly in a dilated left ventricle with reduced ejection fraction. Regional wall motion abnormality in a non-dilated ventricle has a very high specificity for myocardial ischaemia (approaching $100 \%) .^{13}$

Hypertensive heart disease (HHD) was diagnosed in patients with hypertension presenting with symptoms and signs of heart failure, with or without left ventricular (LV) hypertrophy and left atrial enlargement on two-dimensional echocardiography, and Doppler/tissue Doppler evidence of LV diastolic dysfunction in the absence of significant valvular heart disease or regional
Table 1 Sociodemographic characteristics of patients with cardiovascular diseases at six referral/teaching hospitals

\begin{tabular}{|lcr|}
\hline Variables & No. of patients & $\%$ \\
\hline Sex & & \\
\hline Female & 3671 & 58.5 \\
\hline Male & 2604 & 41.5 \\
\hline Age group (years) & & \\
\hline$<5$ & 648 & 10.3 \\
\hline $5-12.9$ & 834 & 13.3 \\
\hline $13-22.9$ & 526 & 8.4 \\
\hline $23-32.9$ & 843 & 13.4 \\
\hline $33-42.9$ & 718 & 11.4 \\
\hline $43-52.9$ & 649 & 10.3 \\
\hline $53-62.9$ & 819 & 13.1 \\
\hline $63-7-42.9$ & 742 & 11.8 \\
\hline$>75$ & 496 & 7.9 \\
\hline Total & 6275 & 100 \\
\hline Age category & & \\
\hline Adult ( $\geq 18$ years) & 4356 & 68.9 \\
\hline Paediatric (<18years) & 1963 & 31.1 \\
\hline Total & 6275 & 100 \\
\hline Residence & & \\
\hline Urban & 3821 & 60.9 \\
\hline Rural & 2454 & 39.1 \\
\hline
\end{tabular}

wall motion abnormality. Primary dilated cardiomyopathy was diagnosed in patients with marked LV dilatation and dysfunction in the absence of significant valvular, structural, or congenital heart disease or arterial hypertension. The institutional review boards of the College of Health Sciences in the respective collaborating universities approved the study.

\section{Statistical methods}

Data were entered into SPSS Version 20 for Windows and were analysed. Median values with interquartile ranges for continuous variables and number and per cent for discrete variables were used to present the data

\section{RESULTS}

Of 7102 patients initially enrolled in the study, $6275(58.5 \%$ females) were included for final analysis. The remaining 827 patients $(11.6 \%)$ were excluded due to lack of echocardiographic reports or proper/reliable echocardiographic reports. Nearly $61 \%$ of the patients were from urban areas. More than two-thirds of the patients $(4356,68.9 \%)$ were adults (age $>18$ years). The median age was 33 years (IQR $14-55$ years). Nearly half of the patients (48.5\%) were from Tikur Anbessa Specialised Hospital, TASH. Table 1 shows the sociodemographic data of the patients included in the study.

Regarding distribution of the types of cardiovascular diseases, valvular heart disease accounted for 2541 (40.5\%) of the patients. Of the cases of valvular heart diseases, 2184 (86\%) were cases of rheumatic heart disease. Pericardial diseases accounted for the smallest proportion. Table 2 shows the distribution of the different types of cardiovascular disorders identified in the study and the corresponding sub-group median ages. In patients $<18$ years of age, congenital heart disease was the most common diagnosis followed by chronic rheumatic valvular heart disease, while valvular heart disease was the most common diagnosis in adults (table 3). Of the congenital heart diseases, ventricular 
Table 2 Distribution of the different cardiovascular diagnosis in patients seen at six referral hospitals

\begin{tabular}{llccc}
\hline Cardiovascular diagnosis & & No. of patients & $\%$ & Median age in years (IQR) \\
\hline & Rheumatic valvular heart disease & 2184 & 34.6 & $25(15-38)$ \\
Valvular heart disease & Other valvular heart disease & 357 & 5.9 & $55(28-68)$ \\
Congenital heart disease & & 1115 & 17.8 & $5(2-10)$ \\
Hypertensive heart disease & & 854 & 13.6 & $55(42-65)$ \\
Ischaemic heart disease & Dilated & 600 & 9.6 & $55(45-65)$ \\
Cardiomyopathy & Hypertrophic & 477 & 7.6 & $45(30-60)$ \\
& Restrictive & 21 & 0.3 & $22(8.75-40)$ \\
Pulmonary hypertension (isolated) & & 7 & 0.1 & $41(35-47.5)$ \\
Miscellaneous & & 255 & 4.1 & $42(26-57)$ \\
Arrhythmia & & 212 & 3.4 & $50(32-60)$ \\
Pericardial diseases & 132 & 2.1 & $55(40-65)$ \\
Total & & 61 & 1.0 & $35(21.5-50)$ \\
\hline
\end{tabular}

septal defect was the most common, accounting for nearly $35 \%$ (table 4).

In those who were excluded due to lack of a reliable echocardiographic report, the suspected pathologies included valvular heart disease (43\%), hypertension with HHD (21\%), ischaemic heart disease (6\%), various congenital heart diseases (8\%), and heart failure secondary to unidentified pathology (22\%). ${ }^{4}$ In patients who were diagnosed with ischaemic heart disease, hypertension was the most common risk factor, being present in $19 \%$ of patients (table 5). The proportions of patients with HHD (16.1\% vs $12.9 \%)$ and ischaemic heart disease $(8.5 \%$ vs $6.1 \%$ ) were higher in urban versus rural areas (see table 6).

Among patients who were candidates for surgical or percutaneous invasive treatments only 3.2\% received invasive treatment.

\section{DISCUSSION}

Our study is the first to look into the spectrum of cardiovascular diseases involving major referral hospitals of the country and patients of all ages. While an ideal study to address the burden and spectrum of cardiovascular diseases is a population-based one, it is prohibitively expensive to conduct such a study in the sub-Saharan setting. The settings included in our study are sites with organised cardiac follow-up clinics and better diagnostic capacities, including echocardiography and electrocardiography. Most cases of suspected cardiovascular diseases across the country are referred to these sites for diagnosis and treatment.

Table 3 Distribution of types of cardiovascular disease in relation to the age of the patients

\begin{tabular}{|c|c|c|}
\hline Types & $\begin{array}{l}\text { Paediatric (<18years } \\
\text { of age) } \%\end{array}$ & $\begin{array}{l}\text { Adult ( } \geqq 18 \text { years of } \\
\text { age) } \%\end{array}$ \\
\hline Valvular heart disease & 30.7 & 43.4 \\
\hline Congenital heart disease & 60.0 & 4.3 \\
\hline Hypertensive heart disease & 3.1 & 18.1 \\
\hline Ischaemic heart disease & 0.0 & 11.5 \\
\hline Cardiomyopathy & 2.8 & 9.7 \\
\hline $\begin{array}{l}\text { Pulmonary hypertension } \\
\text { (isolated) }\end{array}$ & 1.3 & 4.3 \\
\hline Miscellaneous & 1.6 & 4.4 \\
\hline Arrhythmia & 0.1 & 3.1 \\
\hline Pericardial diseases & 0.5 & 1.2 \\
\hline Total & 100 & 100 \\
\hline
\end{tabular}

As our study is a hospital-based study it is possible that referral bias could play an important role.

In our study, chronic rheumatic valvular heart disease was the most common diagnosis. This is not surprising as Ethiopia has one of the highest prevalences of chronic rheumatic heart disease ${ }^{1415}$ with a malignant course even in young children. ${ }^{16}$ As one of the sub-Saharan countries, the risk factors for rheumatic heart disease, such as illiteracy, scarce access to medical care and lack of echocardiography-based screening practices, are prevalent. A recent echocardiography-based school survey involving different regions of the country showed rheumatic heart disease (World Heart Federation criteria) ${ }^{9}$ had a prevalence of 19 cases per 1000 school children aged between $7-18$ years. ${ }^{17}$

Though rheumatic heart disease was the most common diagnosis, other cardiovascular diseases were also common as shown in table 2. The pattern of congenital heart diseases is more or less similar to studies from different parts of the world that were population-based or hospital-based, with ventricular septal defect being the the most common followed by atrial septal defect, patent arterial duct and tetralogy of Fallot, though some of those studies were on newborns. ${ }^{18-20}$ Only a handful of these patients underwent invasive intervention, though most have indications for early surgical or percutaneous intervention. The proportion of congenital heart diseases in our study is higher than in other studies. This is due to the fact that most children diagnosed with or suspected of having congenital heart diseases are referred to and followed up in only a few of these referral centres, especially at Addis Ababa university (Tikur Anbessa)

Table 4 Types of lesions in patients with congenital heart diseases in six referral hospitals

\begin{tabular}{lcc}
\hline Types & No. of patients & $\%$ \\
\hline Ventricular septal defects & 393 & 34.9 \\
\hline Atrial septal defects & 239 & 21.3 \\
\hline Patent ductus arteriosus & 201 & 18.1 \\
\hline Tetralogy of Fallot & 80 & 7.2 \\
\hline Atrioventricular septal defect (variants) & 47 & 4.2 \\
\hline Congenital pulmonary valve stenosis & 43 & 3.8 \\
\hline Coarctation of the aorta & 25 & 2.2 \\
\hline Transposition of the great arteries & 23 & 2.1 \\
Others & 69 & 6.1 \\
\hline Total & 1119 & 100 \\
\hline
\end{tabular}




\begin{tabular}{|c|c|c|c|}
\hline & & No. of patients & $\%$ \\
\hline \multirow[t]{8}{*}{ Risk factors } & None identified & 395 & 63.5 \\
\hline & Arterial hypertension & 118 & 19 \\
\hline & Multiple risk factors & 33 & 5.3 \\
\hline & Diabetes mellitus & 31 & 5.0 \\
\hline & $\begin{array}{l}\text { Family history of ischaemic heart } \\
\text { disease }\end{array}$ & 20 & 3.2 \\
\hline & Smoking & 13 & 2.1 \\
\hline & Dyslipidaemia & 12 & 1.9 \\
\hline & Total & 622 & 100 \\
\hline \multirow[t]{3}{*}{ Gender } & Male & 334 & 53.7 \\
\hline & Female & 288 & 46.3 \\
\hline & Total & 622 & 100 \\
\hline
\end{tabular}

hospital; this is because of the scarcity of paediatric cardiologists or paediatricians trained in paediatric echocardiography in the peripheral hospitals or private clinics. In contrast, a significant proportion of adults are usually followed up at private clinics or they are referred back to the district hospitals once diagnosis is established and treatment started. It is also usual for adults with important comorbidities like hypertension, chronic kidney disease or diabetes mellitus to be followed in other chronic illness care clinics and not referred to the cardiac clinic until they develop overt heart failure symptoms.

Of note is that cardiovascular diseases such as hypertension and ischaemic heart disease, once thought to be diseases of an affluent society, are also common in our settings. This has also been witnessed in other sub-Saharan African countries. $^{21-23}$ People from urban areas had higher proportions of hypertensive and ischaemic heart diseases. These differences may be related to the difference in the distribution of the various cardiovascular risk factors and lifestyles between rural and urban dwellers. The proportion of HHD is more likely to be underestimated in our study as only hypertensive patients with cardiac symptoms are referred to the cardiology clinics, while those without cardiac symptoms are followed at primary healthcare level or other departments such as nephrology clinics. Additionally, the marked overlap of risk factors for hypertensive and ischaemic heart disease may also lead to underestimation of one and overestimation the other. Of the cardiomyopathies, dilated cardiomyopathy was the the most common one accounting for nearly $95 \%$ of the cardiomyopathy cases. We did not see a case of endomyocardial fibrosis in

Table 6 Dsistribution of types of cardiovascular disease in relation to place of residence

\begin{tabular}{lcc}
\hline Types & Urban (\%) & Rural (\%) \\
\hline Valvular heart disease & 34.9 & 44.9 \\
Congenital heart disease & 22.8 & 13.5 \\
Hypertensive heart disease & 16.1 & 12.9 \\
Ischaemic heart disease & 8.5 & 6.8 \\
Cardiomyopathy & 7.4 & 9.1 \\
Pulmonary hypertension (isolated) & 3.5 & 4.0 \\
Miscellaneous & 3.9 & 4.7 \\
Arrhythmia & 1.9 & 2.9 \\
Pericardial diseases & 0.9 & 1.2 \\
\hline Total & 100 & 100 \\
\hline
\end{tabular}

Key messages

What is already known about this subject?

The prevalence of chronic rheumatic heart disease among school children in different parts of Ethiopia has recently been published. Hospital-based studies have also shown that the proportion of cardiac patients is increasing among outpatient visits and hospital admissions.

\section{What does this study add?}

This study shows the spectrum of cardiovascular patients, both paediatric and adult, that were seen in six major referral hospitals located in different parts of the country. Compared with previous hospital-based studies, this study is more representative of the actual pattern of cardiovascular patients.

\section{How might this impact on clinical practice?}

In many sub-Saharan countries, infectious diseases and other poverty-related diseases are given priority and cardiovascular diseases are not given attention. This study may help to influence policymakers to design both preventive and therapeutic strategies, including improved access to basic diagnostic and therapeutic facilities.

our population of patients. Regarding reported risk factors for ischaemic heart disease (table 4), hypertension was the most common and many patients had more than one risk factor. Other risk factors, such as diabetes mellitus, dyslipidaemia, obesity, smoking, etc, were also present. This suggests that these risk factors are on the increase, as seen in other African studies. ${ }^{3}$ Therefore, specific approaches need to be designed to tackle the global risk factors for cardiovascular diseases addressed by the United Nations Declaration, and prevention and control of infections and poverty-related diseases. ${ }^{3}$ It is difficult to determine if there are regional differences in the pattern of cardiovascular diseases, as our study was not methodologically powered for that.

\section{Limitations of the study}

Our study is a hospital-based study and may not be representative of the actual situation in the population/community. Referral bias should also be taken into consideration when interpreting our data. Lack of investigations for definitive diagnosis for ischaemic heart disease is another limitation of the study.

\section{CONCLUSION}

Our study shows that chronic rheumatic valvular heart disease is the most common cardiovascular diagnosis among patients seen at cardiology clinics of six referral/teaching hospitals in the country, followed by congenital heart diseases. Cardiovascular conditions such as hypertensive and ischaemic heart diseases have accounted for a significant proportion of the cases. Therefore, strategies directed towards primary and secondary prevention of acute rheumatic fever as well as prevention of risk factors for hypertension and ischaemic heart disease may need to be strengthened. The numbers of patients who need invasive treatments are also significant and this may need to be considered by health policymakers.

\footnotetext{
Author affiliations

'Department of Internal Medicine, School of Medicine, College of Health Sciences, Addis Ababa University, Addis Ababa, Ethiopia

${ }^{2}$ Department of Pediatrics \& Child Health, School of Medicine, College of Health Sciences, Addis Ababa University, Addis Ababa, Ethiopia
} 
${ }^{3}$ Department of Internal Medicine, St Paul Millennium Medical School, Addis Ababa, Ethiopia

${ }^{4}$ Department of Internal Medicine, School of Medicine, College of Health Sciences, Mekele University, Mekele, Ethiopia

${ }^{5}$ Department of Pediatrics \& Child Health, School of Medicine, College of Health Sciences, Mekele University, Mekele, Ethiopia

${ }^{6}$ Department of Pediatrics \& Child Health, School of Medicine, College of Health Sciences, Jimma University, Jimma, Ethiopia

${ }^{7}$ Department of Internal Medicine, School of Medicine, College of Health Sciences, Jimma University, Jimma, Ethiopia

${ }^{8}$ Department of Internal Medicine, School of Medicine, College of Health Sciences, Gondar University, Gondar, Ethiopia

${ }^{9}$ Department of Internal Medicine, School of Medicine, College of Health Sciences, Hawassa University, Hawassa, Ethiopia

${ }^{10}$ Minneapolis Heart Institute, Minnesota, USA

Acknowledgements We thank the data collectors/nurses at each facility for their efforts and assistance. We also extend our gratitude to all who have been involved in the care of these patients. We would also thank Dr Solomon Shiferaw, Dr Thomas G Allison and Dr Colin Romero for their valuable comments. Last but not least we thank the hospital administration and record personnel for their valuable assistance.

Contributors DY: conceived and designed the study, participated in the data collection, analysis and interpretation and has critically reviewed the manuscript for scientific content and approved the final version. SG: participated in the design of the study, data collection, analysis and interpretation and has critically reviewed the manuscript for scientific content. BA, DM, EG, HB, HT, SB, AH, TH, WD, AHA, EKG, GN, $\mathrm{DMK}, \mathrm{KW}, \mathrm{TE}, \mathrm{KL}$ : participated in the data collection, analysis and interpretation and has read and approved the manuscript in its current form. VKR: participated in the conception and design of the study and interpretation of the data and has critically reviewed the manuscript for scientific content. ET: participated in the design of the study, data collection, interpretation and wrote the manuscript in its current form. All authors have read and approved the manuscript in its current form. All authors agreed to be accountable for all aspects of the work in ensuring that questions related to the accuracy or integrity of any part of the work are appropriately investigated and resolved.

Funding This study was financed by Friends of Children Heart Fund Ethiopia, Minneapolis, USA.

Competing interests None declared.

Patient consent Consent obtained from Guardian.

Ethics approval Institutional Review Boards of 6 universities that participated.

Provenance and peer review Not commissioned; externally peer reviewed.

(c) Article author(s) (or their employer(s) unless otherwise stated in the text of the article) 2017. All rights reserved. No commercial use is permitted unless otherwise expressly granted.

\section{REFERENCES}

1 Sliwa K, Libhaber E, Elliott C, et al. Spectrum of cardiac disease in maternity in a low-resource cohort in South Africa. Heart 2014;100:1967-74.
2 Ngoungou EB, Aboyans $V$, Kouna $P$, et al. Prevalence of cardiovascular disease in Gabon: a population study. Arch Cardiovasc Dis 2012;105:77-83.

3 Mensah GA. Ischaemic heart disease in Africa. Heart 2008:;94:836-43.

4 Akinkugbe 00. Epidemiology of cardiovascular disease in developing countries. J Hypertens 1990;8:5233-8.

5 Damasceno A, Cotter G, Dzudie A, et al. Heart failure in sub-saharan Africa: time for action. J Am Coll Cardiol 2007;50:1688-93.

6 Vedanthan R, Fuster V. Cardiovascular disease in Sub-Saharan Africa: a complex picture demanding a multifaceted response. Nat Clin Pract Cardiovasc Med 2008;5:516-7.

7 Benjamin EJ, Jessup M, Flack JM, et al. Discovering the full spectrum of cardiovascular disease: minority Health Summit 2003: report of the Outcomes Writing Group. Circulation 2005;111:e124-33.

8 Sibai AM, Fletcher A, Hills M, et al. Non-communicable disease mortality rates using the verbal autopsy in a cohort of middle aged and older populations in Beirut during wartime, 1983-93. J Epidemiol Community Health 2001:55:271-6.

9 Reményi B, Wilson N, Steer A, et al. World Heart Federation criteria for echocardiographic diagnosis of rheumatic heart disease-an evidence-based guideline. Nat Rev Cardiol 2012;9:297-309.

10 Gibbons RJ, Balady GJ, Timothy Bricker J, et al. ACC/AHA 2002 guideline update for exercise testing: summary article. J Am Coll Cardiol 2002;40:1531-40.

11 Mendis S, Thygesen K, Kuulasmaa K, et al. World Health Organization definition of myocardial infarction: 2008-09 revision. Int J Epidemiol 2011;40:139-46.

12 Talle M, Anjorin C, Buba F, et al. Spectrum of cardiovascular diseases diagnosed using transthoracic echocardiography: perspectives from a tertiary hospital in NorthEastern Nigeria. Niger J Cardiol 2016;13:39-45.

13 Medina R, Panidis IP, Morganroth J, et al. The value of echocardiographic regional wall motion abnormalities in detecting coronary artery disease in patients with or without a dilated left ventricle. Am Heart J 1985;109:799-803.

14 Oli K, Tekle-Haimanot R, Forsgren L, et al. Rheumatic heart disease prevalence among schoolchildren of an Ethiopian rural town. Cardiology 1992;80:152-5.

15 Oli K, Porteous J. Prevalence of rheumatic heart disease among school children in Addis Ababa. East Afr Med J 1999;76:601-5.

16 Tadele H, Mekonnen W, Tefera E. Rheumatic mitral stenosis in children: more accelerated course in sub-Saharan patients. BMC Cardiovasc Disord 2013;13:95.

17 Yadeta D, Hailu A, Haileamlak A, et al. Prevalence of rheumatic heart disease among school children in Ethiopia: a multisite echocardiography-based screening. Int J Cardiol 2016;221:260-3.

18 Dilber D, Malcić I. Spectrum of congenital heart defects in Croatia. Eur J Pediatr 2010;169:543-50.

19 Ferencz C, Rubin JD, McCarter RJ, et al. Congenital heart disease: prevalence at Livebirth. the Baltimore-Washington Infant Study. Am J Epidemiol 1985;121:31-6.

20 Bosi G, Scorrano M, Tosato G, et al. The Italian Multicentric Study on Epidemiology of Congenital Heart Disease: first step of the analysis. Working Party of the Italian Society of Pediatric Cardiology. Cardiol Young 1999;9:291-9.

21 Ogeng'O JA, Gatonga P, Olabu BO. Cardiovascular causes of death in an east African country: an autopsy study. Cardiol J 2011;18:67-72.

22 Akinboboye $\mathrm{O}$, Idris $\mathrm{O}$, Akinboboye $\mathrm{O}$, et al. Trends in coronary artery disease and associated risk factors in sub-Saharan Africans. J Hum Hypertens 2003;17:381-7.

23 Vorster $\mathrm{HH}$. The emergence of cardiovascular disease during urbanisation of africans. Public Health Nutr 2002;5:239-43. 